\title{
Pure motor hemiplegia, medullary pyramid lesion, and olivary hypertrophy
}

\author{
J. E. LEESTMA ${ }^{1}$ AND A. NORONHA \\ From the Departments of Pathology and Neurology, Northwestern University School of Medicine, \\ Chicago, Illinois, USA
}

SYNOPSIS The case is presented of a 60 years old man who developed sudden right hemiplegia without other accompanying neurological signs and later a spastic hemiparesis. Neuropathological studies indicated an ischaemic lesion of the left medullary pyramid which was accompanied by hypertrophy of the left inferior olivary nucleus. An additional lesion, demyelination of the right gracile tract, is poorly explained. This case represents the second reported instance of pure motor hemiplegia due to a circumscribed lesion in the medullary pyramid and possibly an unique instance of olivary hypertrophy without obvious damage to the central tegmental tract, ipsilateral superior cerebellar peduncle, or contralateral dentate nucleus. The olivary hypertrophy is thought to have arisen from local damage to the termination of the central tegmental fibres at the left inferior olivary nucleus. The question of the development of spasticity in a pure pyramidal tract lesion is discussed.

Fisher and Curry (1965) defined pure motor hemiplegia as a paralysis, complete or incomplete, of the face, arm, and leg on one side unaccompanied by sensory signs, visual field defect, dysphasia, or apractagnosia. In their cases that came to necropsy the syndrome resulted from infarction in the internal capsule or the basis pontis. They stated that it was doubtful that a pyramidal infarction would result in pure motor hemiplegia without other medullary signs. Chokroverty et al. (1975a) reported the first case of an infarction of the medullary pyramid in which hemiplegia was the only sign. We report another case of pure motor hemiplegia due to an ischaemic vascular lesion of the medullary pyramid.

\section{CASE REPORT}

A 60 years old man developed weakness of the right side of his body on 2 November 1973. The onset of weakness was sudden. There was no impairment of

\footnotetext{
1 Address for correspondence and reprint requests: Dr Leestma, Department of Pathology, Northwestern University School of Medicine, 303 East Chicago Avenue, Chicago, Illinois 60611, USA. (Accepted 26 April 1976.)
}

consciousness, headache, convulsion, paraesthesiae, vertigo, diplopia, dysphagia, or visual difficulty. One month before this episode hypertension was noted for the first time. He was in congestive heart failure and was treated with methyldopa, digoxin and frusemide. There was no previous history of transient ischaemic episodes. At the time of examination he was alert, oriented, and there was no mental impairment. The visual fields were intact; the pupils were equal and reacted to light and accommodation. The extraocular movements were normal and there was no nystagmus. Facial sensation was normal. There was a questionable mild right central facial paresis; there was flattening of the right nasolabial fold but no deviation of the angle of the mouth. No dysphagia, palatal or lingual paresis, or palatal myoclonus was noted. The patient had a right hemiplegia which was dense in the arm, less in the right leg with the muscle strength estimated at $4 / 5$. Initially there was flaccidity in the hemiplegic limb but in two weeks this changed to spasticity on the affected side. The reflexes were brisker on the affected side and right ankle clonus was also noted. The plantar responses were extensor on the right and flexor on the left. There were no cerebellar signs, no sensory deficit to touch, pin-prick, vibration, or joint position. Cortical sensory functions were intact. There were bilateral carotid bruits. A brain scan performed two weeks after admission was interpreted as within normal limits. 
The patient was discharged three months later with improvement in the hemiparesis. He was readmitted in September 1974 with gangrene of the lower right leg. An aortogram revealed occlusive vascular disease with complete obstruction of the right femoral artery. An above the knee amputation was done and the patient recovered promptly. He was admitted again in October of 1975 for an inguinal herniorrhaphy. After this procedure he sustained a cardiac arrest and after abortive resuscitation he died.

NECROPSY FINDINGS The general necropsy revealed severe atherosclerosis which involved aorta, carotid arteries, coronary arteries, and peripheral vessels in the extremities. There was a massive acute myocardial infarction affecting the posterior wall of the left ventricle with evidence of old scarring and hypertrophy throughout the left ventricular myocardium. There was an unruptured abdominal aortic aneurysm. Pulmonary oedema and congestion were noted as were other signs of terminal myocardial failure. The stump of the right leg was well healed.

NEUROPATHOLOGICAL EXAMINATION Grossly, the brain was unremarkable; no obvious lesions presented themselves. The leptomeninges were slightly thickened in keeping with the age of the patient and slight cortical atrophy mostly in the frontal lobes was present. The inferior surface of the brain revealed no obvious abnormalities with the circle of Willis containing only scattered atheromatous plaques, signicantly less in amount than were seen in the extracranial vessels. The plaques were found mostly in the internal carotid vessels, scattered along the middle cerebral arteries, at the distal ends of the vertebral arteries and at the proximal and distal ends of the basilar artery. The circle of Willis was normal anatomically with two vertebral arteries of about equal calibre, two posterior communicating and one anterior communicating artery. No obstruction of any vessel was noted. Outwardly the brain stem and cerebellum appeared unremarkable.

Multiple coronal sections of the brain revealed slight cortical atrophy with widening of the sulci and widening of the insula. The ventricles were slightly enlarged. The cortical ribbon was uniform and there were no cerebral softenings. The globus pallidus and putamen contained several small perivascular lacunes, but none was larger than two millimetres. The thalamus was preserved. Cross-sections of the midbrain revealed a pigmented substantia nigra and a patent aqueduct and no lesions. No lesions were found in the pons. However, the most rostral section of the medulla oblongata revealed that the left medullary pyramid was shrunken and of a tan-brown colour compared with the right. The inferior olive also appeared altered and was substantially larger and more distinct than the right. These changes in the olive were seen throughout the medulla. The specimen terminated at the lower medullary level and no spinal cord was available for study. Sections of the cerebellum revealed no abnormality, the dentate nuclei appearing well preserved.

Microscopically, sections stained with haematoxylin and eosin, and Klüver-Barrera stain (Nissl with Luxol Fast Blue) were studied. The sections correlated well with the gross findings. The cerebral cortex was unremarkable. One or two senile plaques were found only in the hippocampus. The small lacunar infarcts in the basal ganglia were all old and the sections of mid-brain and pons showed no lesions and uniform myelinization. The most rostral section of the medullary region (Fig. 1) showed myelin loss in the left pyramid extending to a small extent into the medial lemniscus. There was also some pallor in the myelin of the external arcuate fibres lateral to the left olive. The olive itself showed no alterations apart from the usual accumulation of lipofuscin within the neurones at this level. There was pronounced astrocytosis in the affected pyramid as well as a slight perivascular lymphoid cell infiltrate. Scattered lipid laden macrophages as well as some swollen axons were seen in the pyramid. The opposite pyramid was unremarkable.

In the mid-portion of the medulla at the level of the 12th cranial nerve nucleus (Fig. 2) several changes

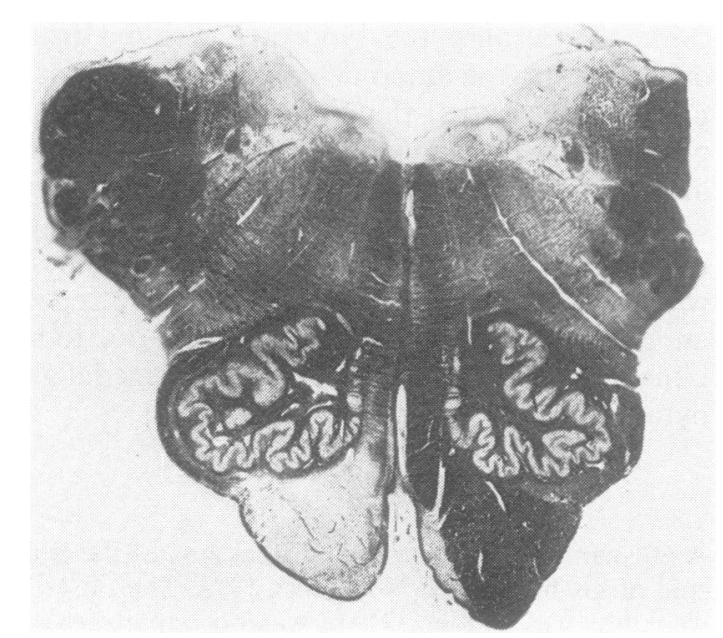

FIG. 1 The most rostral section of medulla stained by the Klüver-Barrera method shows myelin loss in the left pyramid as well as slight pallor laterally and medially. No hypertrophic changes in the inferior olives at this level are noted. 


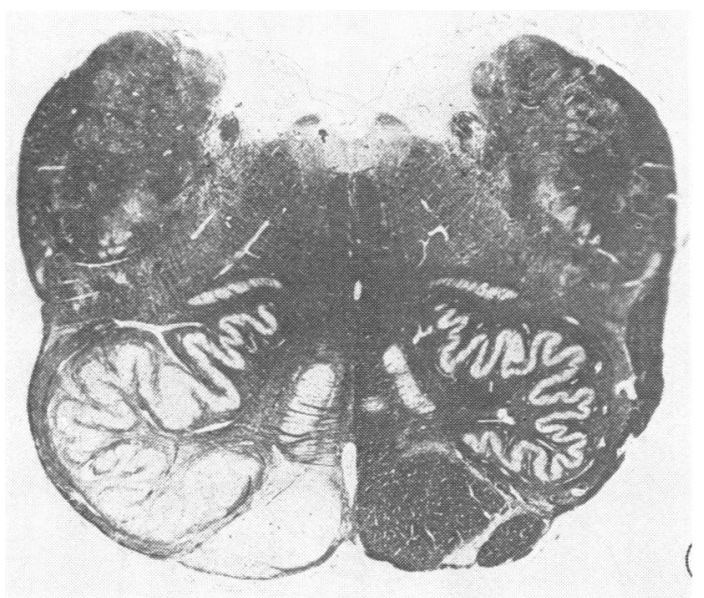

FIG. 2 A mid-section of the medulla reveals the same pyramidal myelin loss as in Fig. 1 but additionally shows hypertrophic changes in the left inferior olivary nucleus. Myelin pallor lateral and medial to the olive is also noted.

were apparent. In addition to the changes previously noted in the pyramid, there was pronounced hypertrophy of the left inferior olivary complex. The olivary neurones were generally swollen, pale, and contained numerous clear vacucles with only occasional cells appearing shrunken and pyknotic. Astrocytosis was also present. In some neurones the cytoplasm appeared to ke refractile and what Nissl substance could be found was compressed into dark specks. The opposite olivary nucleus was normal in appearance as were the 12 th nerve nuclei and other dorsal nuclear masses.

A section of the caudal medulla (Fig. 3) revealed, in addition to the previously observed changes of the pyramid and olive, that the right gracile tract had lost substantial amounts of myelin. Neurones in this region were preserved but there were numerous spheroids, mineralized concretions, and astrocytosis. With the exception of the myelin loss these same changes were seen in the opposite gracile tract and nucleus. The cuneate nucleus showed similar dystrophic changes. There was no inflammatory or phagocytic activity in the right gracile tract and nucleus.

Histological examination of the cerebellum including the dentate nuclei revealed no changes apart from hypoxic alterations which were interpreted as agonal.

The outstanding neuropathological findings in this patient which deserve comment are: degenerative

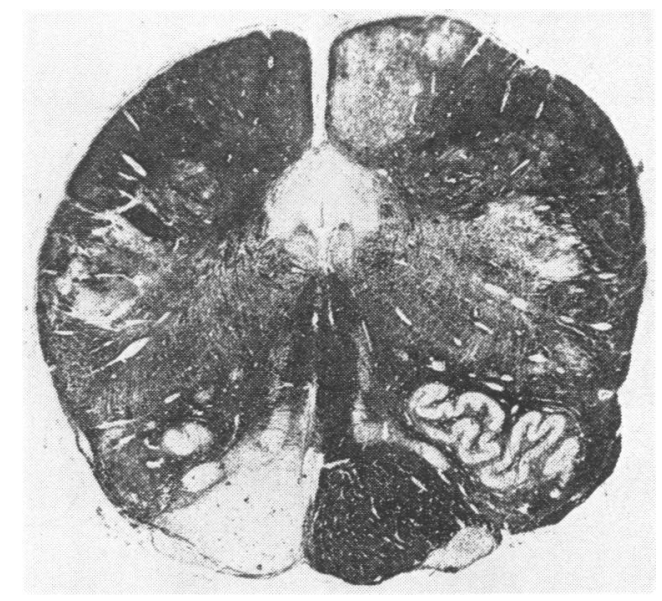

FIG. 3 The most caudal section of medulla illustrates the continued pyramidal tract demyelination, hypertrophy of such olivary elements as are remaining on the left side, and myelin loss in the right gracile nucleus and tract.

alterations in the left medullary pyramid; hypertrophy of the caudal half of the left inferior olivary nucleus; myelin loss in the right gracile tract.

\section{DISCUSSION}

The clinical features in our patient are consistent with the definition of pure motor hemiplegia as described by Fisher and Curry (1965) and similar to such a case as reported by Chokroverty et al. $(1975 a, b)$. A review of the blood supply to the medulla oblongata is necessary to appreciate the possible pathogenesis of the pyramidal and olivary lesions.

The first comprehensive work on the blood supply of the brain stem is the work of Stopford (1916a,b,c). Later investigations on the subject added only slightly to his observations (Foix and Hillemand, 1925; Krayenbühl and Yasargil, 1957). From a review of Stopford's work and examination of several specimens of our own, it is apparent that the blood supply of the medulla is complex and variable. Figure 4 illustrates the blood supply of the ventral medulla. As Foix and Hillemand (1925) pointed out there are medial (paramedian), short and long lateral vessels which arise from the vertebral and anterior spinal 


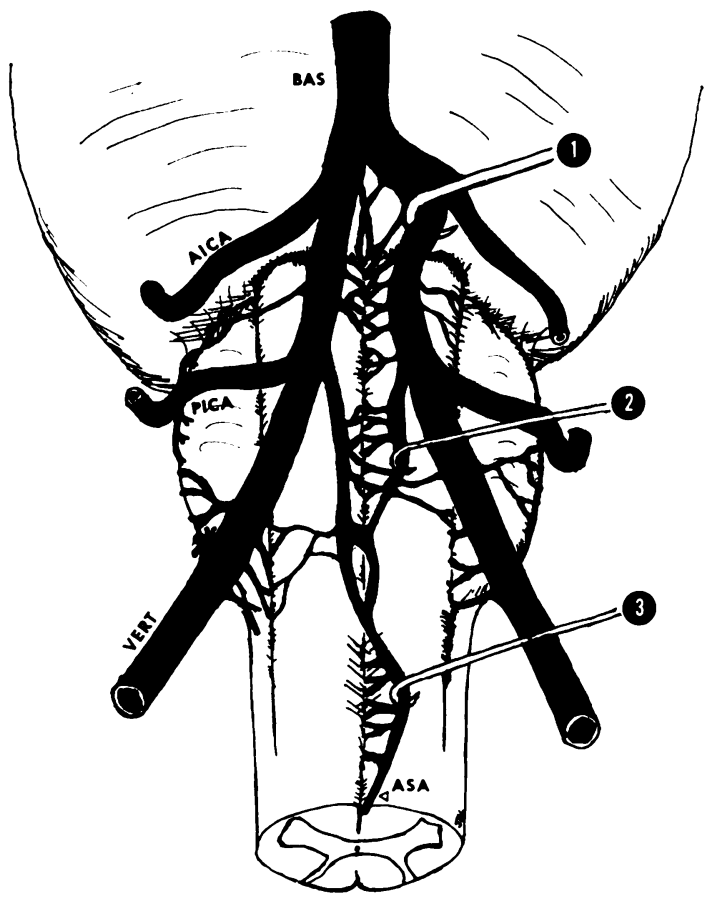

FIG. 4 The vascular supply of the medulla. Two vertebral arteries (VERT) join to form the basilar artery $(B A S)$ giving off two major branches, the posterior inferior cerebellar arteries (PICA) and the anterior inferior cerebellar arteries (AICA). Finer branches, most of which penetrate the midline to supply the pyramids and adjacent structures, occur in three general groups: (I) those arising from the upper vertebral arteries, (2) those which arise at the origin or upper portion of the anterior spinal artery $(s)(A S A)$, and (3) those which arise more caudally along the anterior spinal artery where it is a single vessel.

arteries which supply the ventral and lateral medulla. The rostral median branches arise from the vertebral artery and penetrate the midline between the pyramids, there to ramify and supply the pyramid, medial portion of the olive, medial lemniscus, and other structures which include the floor of the fourth ventricle and the cranial nerve nuclei dorsally near the midline as depicted in Fig. 5. Other more lateral branches supply lateral medulla and reticular formation leaving the restiform body (inferior cerebellar peduncle) and vestibular nuclei to be supplied by branches of the

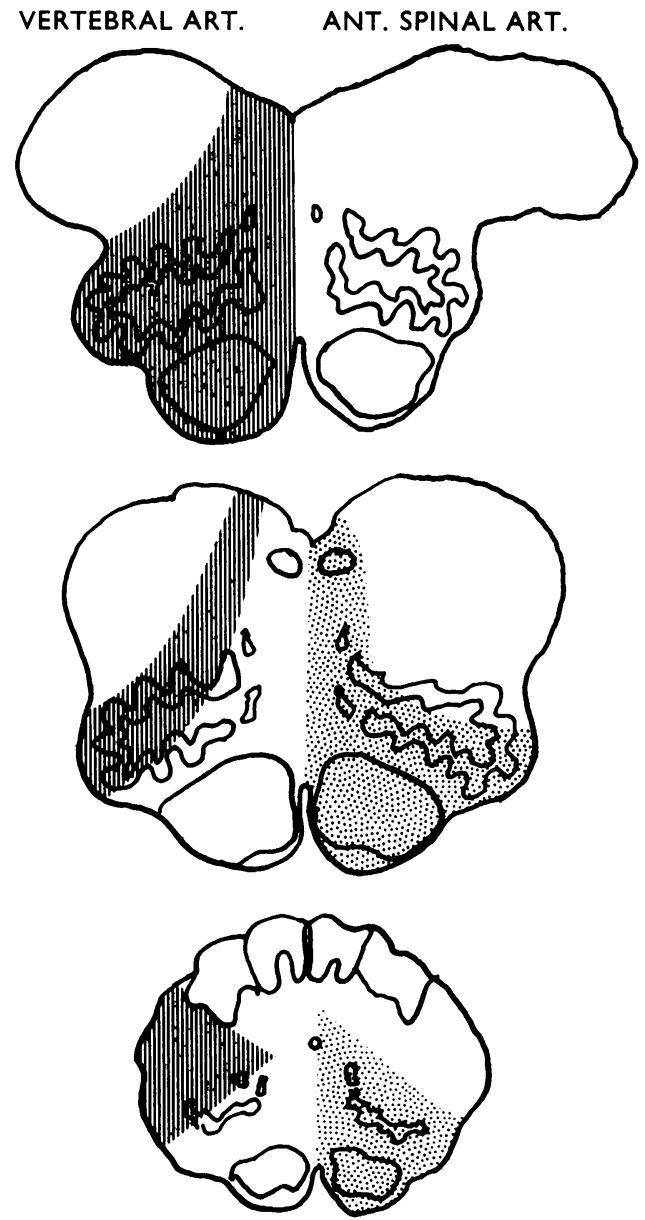

FIG. 5 The territories of supply of the vertebral arterial branches and the small branches of the anterior spinal artery in the medulla. The supply of the posterior inferior cerebellar artery and its branch, the posterior spinal artery are not shown but supply the remaining unshaded or unspotted regions (after Stopford, 1916a,b).

posterior inferior cerebellar artery. At this most rostral level, the junction between pons and medulla, there is usually no circulatory contribution made by the anterior spinal artery. At mid-medullary levels a narrow territory is supplied by the vertebral artery which runs from the region lateral to the dorsal motor nucleus of the vagus through the inferior olive laterally. The 
remaining ventral and midline structures are supplied by similar median and short lateral branches of vessels arising from the anterior spinal artery as shown in the same figure. More dorsal structures are supplied by the posterior spinal artery and branches of the posterior inferior cerebellar artery.

In the caudal medulla, anterior spinal artery branches supply the pyramid and median structures as well as some ventral lateral regions. There is some territorial overlap between the more lateral supply of the vertebral arterial branches and the posterior spinal artery territory. It is possible that a 'watershed zone' exists in the region jointly supplied by small vessels arising from the vertebral artery and those of the anterior spinal artery at upper-mid medullary levels. The existence of such a 'watershed' might be important to the pathogenesis of ischaemic lesions in this region such as the case reported above.

PYRAMIDAL LESION A discrete lesion as described above is unusual but has been described in one recent report (Chokroverty et al., 1975a) and in at least two previous reports in the older literature. One of these was probably of atherosclerotic origin in which the lower medulla at the level of the pyramidal decussation was affected by a discrete lesion (Stopford, 1916c), and another in which syphilitic vasculitis most probably resulted in ischaemia of the pyramid and adjacent structures, much as in our case except that there is no suggestion of syphilis in the present patient (Lhermitte and Trelles, 1933). In this latter case, the pyramidal lesion was accompanied by olivary hypertrophy. We surmise that some impairment of circulation to the left pyramid of our patient occurred in 1973 which may have been in the form of a migrating thromboembolus, in situ thrombosis, hypotensive episode, or all three. The area deprived of blood supply was in the territory served by the medial penetrating branches of either the vertebral or anterior spinal arteries of the left side. It is likely that a long-standing total occlusion did not occur, for if it had, the result would have been a cavitary lesion of larger proportions probably not limited to the small area observed in this case. In addition, there would have been more extensive neuronal damage than was observed here where arcuate nuclei were preserved and the olive was still viable. The myelin loss below the rostral medulla most probably represents a descending degeneration which, if the spinal cord had been available for study, would have shown a continuation of the degeneration downward into the right corticospinal tract. The inflammatory infiltrate and persistent macrophage response is typical for old infarctive or ischaemic lesions and in degenerating tracts. There is no suggestion in the material or in the rest of the case to suggest an inflammatory or infectious aetiology for the brain stem pathology.

FUNCTIONAL IMPLICATIONS OF PYRAMIDAL LESION According to classical concepts, in a pyramidal tract lesion one expects to observe a spastic (hypertonic) paralysis of the affected extremity after the immediate period of neural shock abates; however, it appears that when neurosurgical interruption of the pyramidal tract in man or in monkeys occurs the opposite is observed, or, at best, variable results have been obtained (Beck and Chambers, 1970; Gilman and Marco, 1971). In the few studies involving man, most notably the report of Bucy et al. (1964) where the pyramidal tract was interrupted at the level of the cerebral peduncle, after a period of recovery the patient moved quite well with no important signs of hypertonia with the possible exception of Babinski's sign in the affected lower extremity. It appears then that pure lesions of the pyramidal tract do not produce a spastic type of paralysis in man or in the monkey. The present case must be somewhat of a rarity in this respect as a rather pure lesion seems to be present in the medullary pyramid and yet the patient suffered many of the accepted signs (Landau, 1969) of a hypertonic paralysis. A re-examination of the other reports of similar discrete lesions in the medulla in man (Chokroverty and Rubino, 1975a,b) indicates that in these cases the patients' affected extremities were more hypotonic than hypertonic, though some slight increase in tonicity and a Babinski's sign was observed. Admittedly, a detailed clinical observation was not available on our patient, but from the records available to us many of the criteria for spasticity were met.

Why then in the face of other human material of a similar type where hypotonicity or the lack of hypertonic signs were noted does our case exhibit signs of spasticity? Several possibilities exist, but a complete exploration of them, the 
neuroanatomical and neurophysiological controversies which are involved in the question of the mechanism for spasticity, and the anatomy of the pyramidal tract and its function are beyond the scope of this paper.

Briefly, however, it is possible that anatomical variations played a part in the development of symptoms in this man as it is known that considerable variations exist in the anatomy of the pyramidal tract in man (Nyberg-Hansen and Rinvik, 1963). It could be that most of this man's motor connections with the spinal cord were interrupted by the lesion, with virtually no contribution from the opposite pyramidal tract existing. This lesion is quite distinct but there is some extension into the medial leminiscus not to mention the obvious involvement of the inferior olive on the left side and the myelin loss in the gracile tract on the right side (which is poorly explained). It is possible that these lesions might further contribute to the already heavily deafferented motor horn, thus releasing it to local influences which are said to be the basis for spasticity in instances such as spinal cord injury where more than simple motor interruption occurs (Bucy et al., 1964; Dimitrijevic and Nathan, 1967; Landau, 1969; Gilman and Marco, 1971). This hypothesis is further strengthened by the recent report of Chokroverty (Chokroverty and Rubino, 1975b) that in such discrete pyramidal lesions as our case there may be subtle sensory dysfunction as well.

OLIVARY LESION The subject of olivary hypertrophy has been studied for many years with several reports antedating the turn of the century. Thesc older references can be found in a recent article (Horoupian and Wisniewski, 1971). Since that time several reports have dealt with the phenomenon itself and in relation to palatal myoclonus which sometimes occurs with olivary hypertrophy (Marie and Foix, 1913; Lhermitte and Trelles, 1933; Davison et al., 1936; Gautier and Blackwood, 1961; Aberfeld, 1966; Lapresle and BenHamida, 1970; Sohn and Levine, 1971). From these accounts it is apparent that olivary hypertrophy can occur when there are interruptions of the ipsilateral central tegmental tract, or when this tract is intact but when the contralateral dentate nucleus in the cerebellum has been destroyed or its fibres interrupted. Lesions of the superior cerebellar peduncle can similarly produce the lesion on the ipsilateral olive (Vuia and Rothemund, 1971). It appears that this unusual reaction on the part of the olive results from significant deafferentation at some distance from it or by lesions in its immediate vicinity (Aberfeld, 1966). Interruption of efferent olivary fibres is not known to produce hypertrophy but induces atrophy (Aberfeld, 1966; Sohn and Levine, 1971).

The anatomical connections of the inferior olivary nuclei in man are based on relatively few studies with most data having been extrapolated from studies in cat and to a lesser extent from monkey and other animals. In this system such animal studies probably have only limited relevance to man (Scheibel and Scheibel, 1955), as pathways demonstrated by many workers in cats projecting to the olives have not been substantiated in man (Verhaart and Voogd, 1962; Lapresle and BenHamida, 1970).

The inferior olive in man probably receives input from a number of sources both above and below it, but the major contribution comes from the contralateral dentate nucleus of the cerebellum passing into the brain stem by way of the brachium conjunctivum (superior cerebellar peduncle) probably collecting some fibres from the red nucleus or its vicinity and crossing the midline within the commissure of Wernekink at that level. From here the fibres descend within the central tegmental tract to enter the inferior olive over a broad front superiorly and laterally (Gautier and Blackwood, 1961; Verhaart and Voogd, 1962; Crosby et al., 1962; Lapresle and BenHamida, 1970). There is a rich intercommunication between the olives on both sides by means of a mass of crossing fibres which pass through the medial lemniscus (Scheibel and Scheibel, 1955; Crosby et al., 1962).

Output from the olivary nuclei also varies in many respects from species to species but the major outflow in man is probably to the cerebellar cortex with lesser connections going to the nearby reticular formation and to the spinal cord (Holmes and Stewart, 1908; Crosby et al., 1962).

From an examination of Fig. 1 it is evident at the highest medullary level shown that there is some myelin loss laterally in the external arcuate fibres of the left olive, though there are no changes of olivary hypertrophy at this level. Likewise, there is pallor in the region between the two 
olivary nuclei. Similar changes, though more developed, are noted in the mid-section of the medulla in Fig. 2. Here the hypertrophic changes are quite distinct. The question now arises, what is the cause for the hypertrophic change observed? The most satisfactory explanation is that the central tegmental fibres which entered the left olive by way of the external arcuate bundle were damaged by the ischaemic process in the region, with damage most severe in the lower portion of the medulla. Since tegmental fibres appear to be the most important for maintenance of the olive, a large number of these fibres at the lower level must have been interrupted. It is likely that many decussating fibres were also damaged by the lesion but these must have been of lesser importance to the opposite olive as no changes of hypertrophy or neuronal reaction were noted there. Such regional lesions are thought to be important to the development of hypertrophy as has been pointed out by Aberfeld (1966).

The cellular alterations in the neurones of the left olive consisting of swelling, chromatolysis, vacuolation, occasional shrinkage, and filamentous hypertrophy are typical and have been described elsewhere (Lhermitte and Trelles, 1933; Gautier and Blackwood, 1961; Horoupian and Wisn:ewski, 1971; Sohn and Levine, 1971). A unique feature illustrated in this case is that hypertrophy of the olive can arise from a local lesion and not by a major lesion rostral to it as is usually the case.

The absence of palatal myoclonus is frustrating but its absence in the presence of known lesions of the central tegmental tract with and without olivary hypertrophy has been noted, though unexplained, by others (Lhermitte and Trelles, 1933; Davison et al., 1936). From an anatomical point of view this problem is a nagging one and only further experimental work will resolve this enigma.

GRACILE TRACT DEMYELINATION We are at a loss to explain adequately the myelin loss in this tract and nucleus on the right. The high leg amputation accomplished several months before death ablated some sensory output into the ipsilateral gracile tract but, in the absence of either a root or dorsal root ganglion lesion, ascending degeneration would ordinarily not be expected (Norton, 1969). The changes seen in the tract and nucleus do not resemble those of an infarct or ischaemic lesion, though this latter possibility should be considered. Other changes in the gracile and cuneate nuclei of the caudal medulla include spheroids, mineralized concretions, and gliosis which can be regarded as accompaniments of the aging process as documented by others (Brannon et al., 1967). The fact that there were no clinical signs of sensory disturbance (touch and position) on the right side in view of some myelin loss in the right inferior medial lemniscus is also curious. This region would have been expected to produce some impairment of these sensory modalities in the right leg (Crosby et al., 1962) due to somatotopic localizations in the medial lemniscus. It is possible that, with the severe peripheral vascular disease present, this relatively subtle change was not appreciated. On the other hand it is possible that the medullary lesion had not yet expanded at the time of the careful neurological examination into that area to cause symptoms or signs. Later on, with the amputation all chances of documenting such a possible deficit were lost with the leg. That subtle sensory deficits can occur in cases of pure motor hemiplegia and be clinically unnoted is described in a recent publication by Chokroverty and Rubino (Chokroverty and Rubino, 1975b).

This case represents an unusual combination of clinical and anatomical problems which may be unique. It further illustrates the value but frustration inherent in human neurological material in that, had the character of the lesion been appreciated sooner, more elegant neuroanatomical techniques might have been applied and more might have been learned from the unusual lesions in this case.

\section{REFERENCES}

Aberfeld, D. C. (1966). The hypertrophic degeneration of the olives. Acta Neurologica Scandinavica, 42, 296-306.

Beck, C. H., and Chambers, W. W. (1970). Speed, accuracy and strength of forelimb movement after unilateral pyramidotomy in rhesus monkeys. Journal of Comparative and Physiological Psychology, 70, 1-22.

Brannon, W., McCormick, W., and Lampert, P. W. (1967). Axonal dystrophy in the gracile nucleus of man. Acta Neuropathologica, 9, 1-6.

Bucy, P. C., Keplinger, J. E., and Siqueira, E. B. (1964). Destruction of the 'pyramidal tract' in man. Journal of Neurosurgery, 21, 385-398. 
Chokroverty, S., and Rubino, F. A. (1975b). 'Pure' motor hemiplegia. Journal of Neurology, Neurosurgery, and Psychiatry, 38, 896-899.

Chokroverty, S., Rubino, F. A., and Haller, C. (1975a). Pure motor hemiplegia due to pyramidal infarction. Archives of Neurology (Chic.), 32, 647-648.

Crosby, E. C., Humphrey, T., and Lauer, E. W. (1962). Correlative Anatomy of the Nervous System. Macmillan: New York.

Davison, E., Riley, H. A., and Brock, S. (1936). Rhythmic myoclonus of the muscle of the palate, larynx, and other regions. Bulletin of the Neurological Institute N.Y., 5, 94-126.

Dimitrijevic, M. R., and Nathan, P. W. (1967). Studies of spasticity in man. I. Some features of spasticity. Brain, 90, 1-28.

Fisher, C. M., and Curry, H. B. (1965). Pure motor hemiplegia of vascular origin. Archives of Neurology (Chic.), 13, 30-44.

Foix, C., and Hillemand, P. (1925). Les artères de l'axe encéphalique jusqu'au diencéphale inclusivement. Revue Neurologique, 2, 706-739.

Gautier, J. C., and Blackwood, W. (1961). Enlargement of the inferior olivary nucleus in association with lesions of the central tegmental tract or dentate nucleus. Brain, 84, 341-360.

Gilman, S., and Marco, L. A. (1971). Effects of medullary pyramidotomy in the monkey. I. Clinical and electromyographic abnormalities. Brain, 94, 495-514.

Holmes, G., and Stewart, T. G. (1908). On the connection of the inferior olives with the cerebellum in man. Brain, 31, 125-137.

Horoupian, D. S., and Wisniewski, H. (1971). Neurofilamentous hyperplasia in inferior olivary hypertrophy. Journal of Neuropathology and Experimental Neurology, 30, 571-582.

Krayentühl, H., and Yasargil, M. G. (1957). Die Vaskulären Erkrankungen in Gebiet der Arteria Vertebralis und Arteria Basialis. Eine anatomische und pathologische, klinische und neuroradiologische Studie. Thieme: Stuttgart.
Landau, W. M. (1969). Spasticity and rigidity. In Contemporary Neurology Series, Vol. 6, Recent Advances in Neurology. Edited by F. Plum. Davis: Philadelphia.

Lapresle, J., and BenHamida, M. (1970). The dentatoolivary pathway. Somatotopic relationship between the dentate nucleus and the contralateral inferior olive. Archives of Neurology (Chic.), 22, 135-143.

Lhermitte, J., and Trelles, J. O. (1933). L'hypertrophie des olives bulbaires. L'Encéphalie, 28, 588-600.

Marie, P., and Foix, C. (1913). Sur la dégénération pseudohypertrophique de l'olive bulbaire. Revue Neurologique, 26, 48-52.

Norton, A. C. (1969). The Dorsal Column System of the Spinal Cord. Its Anatomy, Physiology, Phylogeny and Sensory Function. UCLA Brain Information Service, National Institute of Health-NINDS: Bethesda, Md.

Nyberg-Hansen, R., and Rinvik, E. (1963). Some comments on the pyramidal tract with special reference to its individual variations in man. Acta Neurologica Scandinavica, 39, 1-30.

Scheibel, M. E., and Scheibel, A. B. (1955). The inferior olive. A Golgi study. Journal of Comparative Neurology, 102, 77-131.

Sohn, D., and Levine, S. (1971). Hypertrophy of the olives: A report on 43 cases. In Progress in Neuropathology, Vol. 1. Edited by H. M. Zimmerman. Grune and Stratton: New York.

Stopford, J. S. B. (1916a). The arteries of the pons and medulla oblongata. Journal of Anatomy and Physiology, 50, 131-164.

Stopford, J. S. B. (1916b). The arteries of the pons and medulla oblongata: Part 2. Journal of Anatomy and Physiology, 50, 255-280.

Stopford, J. S. B. (1916c). The arteries of the pons and medulla oblongata: Part 3. Journal of Anatomy and Physiology, 51, 250-277.

Verhaart, W. J. C., and Voogd, J. (1962). Hypertrophy of the inferior olives in the cat. Journal of Neuropathology and Experimental Neurology, 21, 92-104.

Vuia, O., and Rothemund, E. (1971). L'hypertrophie de l'olive bulbaire dans le syndrome apallique post-traumatique. Revue Neurologique, 125, 373-386. 